\title{
Oral D-galactose supplementation in PGM1-CDG
}

\author{
Sunnie Yan-Wai Wong, BA ${ }^{1}$, Therese Gadomski, BS ${ }^{1}$, Monique van Scherpenzeel, $\mathrm{PhD}^{2}$, \\ Tomas Honzik, MD, PhD ${ }^{3}$, Hana Hansikova, $\mathrm{PhD}^{3}$, Katja S. Brocke Holmefjord, MD ${ }^{4}$, Marit Mork, $\mathrm{MD}^{4}$, \\ Francis Bowling, $\mathrm{MD}, \mathrm{PhD}^{5}$, Jolanta Sykut-Cegielska, $\mathrm{MD} \mathrm{PhD}^{6}$, Dieter Koch, $\mathrm{MD}^{7}$, \\ Jozef Hertecant, $\mathrm{MD}^{8}$, Graeme Preston, $\mathrm{MS}^{1}$, Jaak Jaeken, MD, $\mathrm{PhD}^{9}$, Nicole Peeters, $\mathrm{MS}^{1}$, \\ Stefanie Perez, MS ${ }^{1}$, David Do Nguyen, BA ${ }^{1}$, Kea Crivelly, MS ${ }^{1}$, Tim Emmerzaal, MS ${ }^{10}$, \\ K. Michael Gibson, $\mathrm{PhD}^{11}$, Kimiyo Raymond, $\mathrm{MD}^{12}$, Nurulamin Abu Bakar, $\mathrm{PhD}^{2}$, \\ Francois Foulquier, $\mathrm{PhD}^{13}$, Gernot Poschet, $\mathrm{PhD}^{14}$, Amanda M Ackermann, MD, PhD ${ }^{15}$, Miao He, $\mathrm{PhD}^{16}$, \\ Dirk J Lefeber, PhD ${ }^{2}$, Christian Thiel, PhD ${ }^{17}$, Tamas Kozicz, MD, PhD ${ }^{1,10}$ and Eva Morava, MD, PhD ${ }^{1}$
}

\begin{abstract}
Purpose: Phosphoglucomutase-1 deficiency is a subtype of congenital disorders of glycosylation (PGM1-CDG). Previous case reports in PGM1-CDG patients receiving oral D-galactose (D-gal) showed clinical improvement. So far no systematic in vitro and clinical studies have assessed safety and benefits of D-gal supplementation. In a prospective pilot study, we evaluated the effects of oral D-gal in nine patients.
\end{abstract}

Methods: D-gal supplementation was increased to $1.5 \mathrm{~g} / \mathrm{kg} / \mathrm{day}$ (maximum $50 \mathrm{~g} /$ day) in three increments over 18 weeks. Laboratory studies were performed before and during treatment to monitor safety and effect on serum transferrin-glycosylation, coagulation, and liver and endocrine function. Additionally, the effect of D-gal on cellular glycosylation was characterized in vitro.

Results: Eight patients were compliant with D-gal supplementation. No adverse effects were reported. Abnormal baseline results (alanine transaminase, aspartate transaminase, activated partial thromboplastin time) improved or normalized already using $1 \mathrm{~g} / \mathrm{kg} /$ day D-gal. Antithrombin-III levels and transferrin-glycosylation showed significant improvement, and increase in galactosylation and whole glycan content. In vitro studies before treatment showed $N$-glycan hyposialylation, altered $O$-linked glycans, abnormal lipid-linked oligosaccharide profile, and abnormal nucleotide sugars in patient fibroblasts. Most cellular abnormalities improved or normalized following D-gal treatment. D-gal increased both UDP-Glc and UDP-Gal levels and improved lipid-linked oligosaccharide fractions in concert with improved glycosylation in PGM1-CDG.

Conclusion: Oral D-gal supplementation is a safe and effective treatment for PGM1-CDG in this pilot study. Transferrin glycosylation and ATIII levels were useful trial end points. Larger, longer-duration trials are ongoing.

Genet Med advance online publication 15 June 2017

Key Words: coagulation; glycomics; LLO; glycosylation; phosphoglucomutase 1

\section{INTRODUCTION}

Congenital disorders of glycosylation (CDGs) are defects in glycoprotein and glycolipid synthesis. ${ }^{1}$ Phosphoglucomutase 1 deficiency (PGM1-CDG; MIM 612941) is a recently characterized CDG with orofacial malformations and multisystem involvement including cardiomyopathy, coagulopathy, endocrinopathies, and hepatopathy. ${ }^{2-4}$ These clinical features have been attributed to abnormal protein glycosylation. Additionally, patients suffer from recurrent hypoglycemia and myopathy, which is thought to occur by insufficient glycogen mobilization due to PGM1 deficiency. ${ }^{5,6}$

PGM1 is required for the interconversion of glucose 1-phosphate and glucose 6-phosphate, and is a key enzyme in glycolysis, glycogenesis, and glycogenolysis. ${ }^{7,8}$ Although the exact mechanism remains unclear, PGM1 deficiency has been associated with abnormal intracellular levels of glucose and galactose metabolites, as well as reduced assembly and remodeling of $N$-linked glycans. These metabolic disturbances

\footnotetext{
${ }^{1}$ Hayward Genetics Center, Tulane University School of Medicine, New Orleans, Louisiana, USA; ${ }^{2}$ Department of Neurology, Translational Metabolic Laboratory, Donders Institute for Brain, Cognition, and Behavior, Radboudumc, Nijmegen, The Netherlands; ${ }^{3}$ Department of Pediatrics and Adolescent Medicine, First Faculty of Medicine, Charles University in Prague and General University Hospital in Prague, Prague, Czech Republic; ${ }^{4}$ Department of Pediatric Habilitation, Stavanger University Hospital, Stavanger, Norway; ${ }^{5}$ Biochemical Diseases, Mater Children's Hospital, South, Brisbane, Queensland, Australia; ${ }^{6}$ Screening and Metabolic Diagnostics Department, The Institute of Mother and Child, Warsaw, Poland; ${ }^{7}$ Pediatric Cardiology, Bergisch Gladbacher Koln, Koln, Germany; ${ }^{8}$ Genetics/Metabolics Service, Tawam Hospital, Al Ain, United Arab Emirates; ${ }^{9}$ Department of Pediatrics, University Hospitals Leuven, Leuven, Belgium; ${ }^{10}$ Department of Anatomy, Radboud University Medical Centre, Nijmegen, The Netherlands; ${ }^{11}$ Washington State University College of Pharmacy, Spokane, Washington, USA; ${ }^{12}$ Division of Laboratory Genetics, Department of Laboratory Medicine and Pathology, Mayo Clinic, Rochester, Minnesota, USA;

${ }^{13}$ University Lille, CNRS, UMR 8576-UGSF-Unité de Glycobiologie Structurale et Fonctionnelle, Lille, France; ${ }^{14}$ Centre for Organismal Studies (COS), University of Heidelberg, Heidelberg, Germany; ${ }^{15}$ Division of Endocrinology and Diabetes, The Children's Hospital of Philadelphia, Philadelphia, Pennsylvania, USA; ${ }^{16}$ Palmieri Metabolic Disease Laboratory, The Children's Hospital of Philadelphia, Philadelphia, Pennsylvania, USA; ${ }^{17}$ Center for Child and Adolescent Medicine, Kinderheilkunde I, University of Heidelberg, Heidelberg, Germany. Correspondence: Eva Morava (emoravakozicz@tulane.edu)

The first two authors contributed equally to this work.

The last two authors contributed equally to this work.

Submitted 23 November 2016; accepted 2 March 2017; advance online publication 15 June 2017. doi:10.1038/gim.2017.41
} 
are thought to be responsible for both the missing and the truncated glycans detected in serum by transferrin isoform analysis. There is a lack of whole glycans and reduction of galactose units and terminal sialic acids in truncated glycans in PGM1-CDG, demonstrated by quadrupole time-of-flight mass spectrometry. ${ }^{3}$

In a landmark study, we reported on improved glycosylation with galactose supplementation in PGM1-CDG. ${ }^{3}$ In vitro studies in skin fibroblast cell lines derived from three patients showed improvement in protein glycosylation following D-galactose (D-gal) treatment. Additionally, in six patients who received dietary supplementation of D-gal, both transferrin glycosylation and total serum $\mathrm{N}$-glycome showed improvement within two weeks. Oral lactose supplements had minimal effect on transferrinisoelectric focusing, and high dietary lactose content did not affect clinical outcome. While improvements were documented in a few patients ingesting D-gal, a systematic clinical study has yet to assess the effect of oral galactose supplementation on other features of PGM1CDG and establish the extent of clinical improvement.

\section{MATERIALS AND METHODS}

\section{Study design}

Nine patients with PGM1 deficiency were enrolled in a prospective pilot-study (Supplementary Files online,
ClinicalTrials.gov NCT02955264). The primary endpoint was short-term safety and tolerability of oral D-gal supplementation and a major goal was to identify physiological biomarkers that are responsive to D-gal supplementation in a heterogeneous genetic background. The secondary endpoints were the restoration of the plasma glycan subfractions, monitored via glycomics (Figure 3, Supplementary Table 3), and normalization of antithrombin III activity and serum alanine transaminase (ALT) levels.

Patient data, mutations, and PGM1 enzyme activity are reported in Table 1, clinical features in Supplementary Table 1.

Additionally, we studied the biological effect of D-galactose in vitro in patient skin fibroblasts.

\section{Three escalating doses of $\mathrm{D}$-gal over 18 weeks}

Participants in this pilot study received oral D-gal supplementation added to the regular diet for 18 weeks. D-gal (D-GALACTOSE or Galaxtra; Supplementary Files) intake was increased over the study period in increments to avoid gastrointestinal side effects; weeks 0-6 (T0-T1), $0.5 \mathrm{~g} / \mathrm{kg} /$ day; weeks $6-12$ (T1-T2), $1.0 \mathrm{~g} / \mathrm{kg}$ per day; weeks 12-18 (T2-T3), $1.5 \mathrm{~g} / \mathrm{kg}$ per day (Supplementary Files, Supplementary Figure Sa). The maximum daily oral dose of D-gal any patient received was $50.0 \mathrm{~g}$, an amount that is within the recommended daily maximum intake and

Table 1 Study participants and their cDNA mutations and the respective amino acid changes

\begin{tabular}{|c|c|c|c|c|c|c|}
\hline Patient & Sex & $\mathrm{Age}^{\mathrm{a}}$ & $\begin{array}{l}\text { cDNA mutation } \\
\text { (NM_002633.2) }\end{array}$ & $\begin{array}{l}\text { Amino acid change } \\
\text { (NP_002624.2) }\end{array}$ & Genotype & $\begin{array}{c}\text { PGM1 enzyme activity in } \\
\text { cultured skin fibroblasts } \\
\text { ( } \% \text { of controls) }\end{array}$ \\
\hline $1^{b}$ & $\mathrm{~F}$ & 21 years & c. $1264 C>T$ & p.R422W & Heterozygous compound & 0 \\
\hline \multirow[t]{2}{*}{$2^{c}$} & M & 11 years & c. $1010 C>T$ & р.T337M & Heterozygous compound & 5 \\
\hline & & & C. $1508 \mathrm{G}>\mathrm{A}$ & p.R503Q & missense & \\
\hline $3^{d}$ & $\mathrm{~F}$ & 19 years & c. $988 \mathrm{G}>\mathrm{C}$ & p.G330R & Heterozygous compound & $1.3^{\mathrm{d}}$ \\
\hline \multirow[t]{4}{*}{$4^{b}$} & M & 2 years & c.157_158delinsG & p.Q53Gfs*15 & Heterozygous compound & $5^{b}$ \\
\hline & & & c. $1507 C>T$ & p.R503X & nonsense and missense & \\
\hline & & & c. $661 \mathrm{C}>\mathrm{T}$ & p.R221C & & \\
\hline & & & c. $1258 \mathrm{~T}>\mathrm{C}$ & p.Y42OH & & \\
\hline $5^{d}$ & M & 13 years & c. $787 \mathrm{G}>\mathrm{T}$ & p.D263Y & Heterozygous compound & $2.8^{d}$ \\
\hline $8^{d}$ & $\mathrm{~F}$ & 16.5 years & $1507 C>T$ & p.R503X & Homozygous nonsense & 7.7 \\
\hline $9^{b}$ & M & 2 years & C. $112 \mathrm{~A}>\mathrm{T}$ & p.N38Y & Homozygous missense & $3.1^{\mathrm{b}}$ \\
\hline
\end{tabular}

cDNA, complementary DNA; F, female; $M$, male; NA, not available.

PGM1 enzyme activity measurements are included where available. Enzyme activity was assayed in cultured skin fibroblasts derived from patients, except for patient 7 , where the activity was measured in patient blood. PGM1 is present in leukocytes but absent in red blood cells, where PGM2 is the dominant PGM isoenzyme. Although PGM2 is more active as a phosphopentomutase than as a phosphoglucomutase, it has shown to exhibit about $10 \%$ phosphoglucomutase activity in vitro (Maliekal et al. ${ }^{22}$ ). (Tegtmeyer, L.C. et al. ${ }^{3}$ ) (Wong, S.Y.-W. et al. ${ }^{4}$ ).

${ }^{a}$ Age at the time of study enrollment.

${ }^{b}$ Individuals previously reported by Wong et al. ${ }^{4}$

Individuals previously reported by Ondruskova et al. ${ }^{23}$

dindividuals previously reported by Tegtmeyer et al. ${ }^{3}$

ePGM1 enzyme activity measured in blood. 
demonstrated safe. ${ }^{9}$ Dietary assessment (Supplementary Files), clinical evaluation, and laboratory studies were completed every 6 weeks (T0, T1, T2, and T3). Liver transaminases (aspartate transaminase (AST), alanine transaminase (ALT)), creatine kinase, and commonly assayed glycoproteins (TSH, TBG, IGF3BP), and coagulation parameters (factors IX, aPTT, and ATIII), were measured in blood (Supplementary Files). Blood galactose-1-phosphate and urine galactitol were measured to monitor safety. Glycosylation studies included transferrin isoelectric focusing and glycomics in serum by mass spectrometry. Electrocardiography/echocardiography and hepatic ultrasonography were conducted at T0 and T3.

\section{Long-term monitoring}

Patient 1 remained on $\mathrm{D}$-gal $1 \mathrm{~g} / \mathrm{kg} /$ day after the study period as D-gal is the only currently available compassionate treatment for PGM1-CDG. The parameters monitored during the study period, including additional coagulation factor measurements, have been followed as part of routine care (Supplementary Table 2).

\section{Phosphoglucomutase 1 enzyme activity}

Measurements in patient fibroblasts (Supplementary Files) were performed in all patients, except for patient 7 (screening performed in blood).

\section{Glycomics analysis in patient blood at T0 and T3}

Based on serum glycan subfractions, ratios were calculated and compared to reference ranges ${ }^{10}$ (Supplementary Files, Figure 3, Supplementary Table 3).

\section{Statistics}

Quantitative data are presented as the mean \pm standard error of the mean. Repeated measures of analysis of variance was used to determine significant differences between pre- and post-D-gal supplement use. $p \leq 0.05$ was considered significant.

\section{In vitro studies characterizing the effect of D-gal supplementation on glycosylation \\ Skin fibroblast culture}

Fibroblasts were studied from four PGM1-CDG patients (patient 1, 2, 5, and 8) who participated in this prospective pilot study. Additionally, cell lines of two other PGM1-CDG patients were used in some of the in vitro experiments (patient cell-line 2015X and cell-line 2013Y, Table 1).

\section{D-gal supplementation in culture}

D-gal (Sigma-Aldrich) was added to culture media at concentrations $0,0.75,2.0$, or $5 \mathrm{mM}$. The duration of D-gal feeding was $1,4,5$, or 7 days. Culture media was refreshed every 2 days. For lipid-linked oligosaccharide (LLO) and protein-linked oligosaccharide (PLO) measurements $10 \mathrm{mM}$ D-gal was added to a serum-deprived culture medium 1 hour prior to the labeling procedure (Supplementary Files).

\section{PGM1 western blotting and ICAM-1 western blotting}

Western blotting in patients' fibroblasts in the absence and the presence of D-gal was performed in patients $1,2,5,8$, and cell-line 2015X in vivo (Supplementary Files).

\section{Lipid-linked and protein-linked oligosaccharide analysis}

Fibroblasts of patients 1, 2, 8, cell-line 2013Y, and two controls were analyzed with or without D-gal supplementation. ${ }^{11}$

\section{Nucleotide sugar analysis}

Nucleotide sugar analysis for uridine diphosphate (UDP)galactose (UDP-Gal) and UDP-glucose (UDP-Glc) was performed in 1 million skin fibroblast cells derived from controls; patients 1, 2, and 8; and 2013Y. Either 0.75 or $2.0 \mathrm{mM}$ D-gal was added to the culture medium for 1 day or 4 days prior to harvesting by scraping ${ }^{12}$ (Supplementary Files).

\section{Matrix-assisted laser desorption/ionization time-of-flight mass spectroscopy}

Fibroblasts from patient 2 were used for $\mathrm{N}$ - and $\mathrm{O}$-linked glycan analysis with and without $\mathrm{D}$-gal feeding in vitro ${ }^{13}$ (Supplementary Files).

\section{RESULTS}

Escalating dosing of D-gal up to $50 \mathrm{~g} /$ day is safe and tolerated

Of the nine patients, eight patients were compliant with daily oral D-gal supplementation. Protocol violations were reported in two patients (Supplementary Data). Patient 8 was noncompliant, and we excluded her from clinical analysis. Her glycomics analysis was evaluated, however, since her serum was collected throughout the study.

Dietary evaluation reported variable daily dairy intake. No patients consumed more than $0.4 \mathrm{~g} / \mathrm{kg} /$ day dietary lactose (equivalent to $0.2 \mathrm{~g} / \mathrm{kg} /$ day D-gal).

Adverse events were monitored weekly. Aside from gastroenteritis in patient 2 , no clinical or metabolic adverse events were reported to be associated with incremental increase in D-gal intake. No serious adverse events were reported.

Safety parameters were normal and remained stable during the trial, with no patients experiencing increased galactitol excretion in urine. Galactose-1-phosphate levels were successfully monitored in patients $1,2,3,5,7$, and 9 , and no abnormalities were found at T2 and T3 (data not shown). No structural cardiac or liver changes were noted at study endpoint (data not shown).

\section{Liver function: improvement in AST and normalization of ALT}

Baseline AST of all patients was 3- to 30-fold higher than the upper limit of normal (Figure 1a). AST moderately declined at 6 weeks, and at 12 weeks, AST declined by $23 \%$ to more than 10 -fold. With the exception of patient 2, AST remained relatively stable, and while it did not normalize at 18 weeks, it 
a
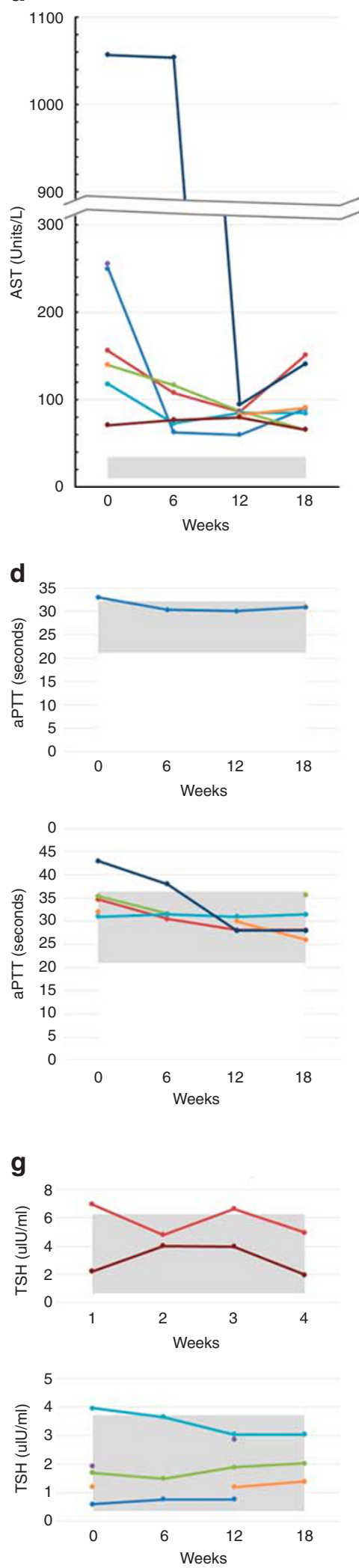

b



e

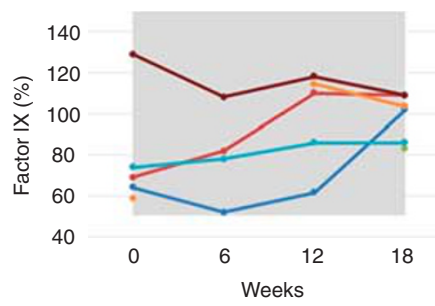

h



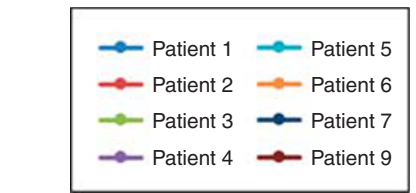

C

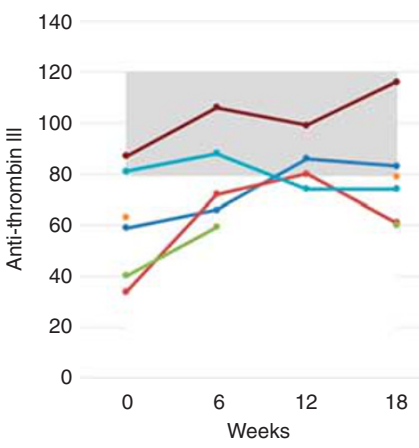

f
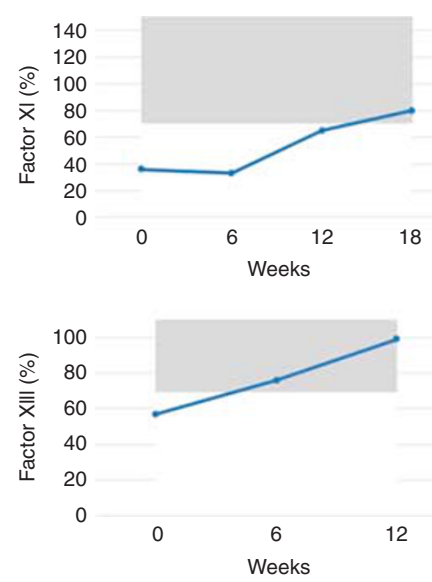

i

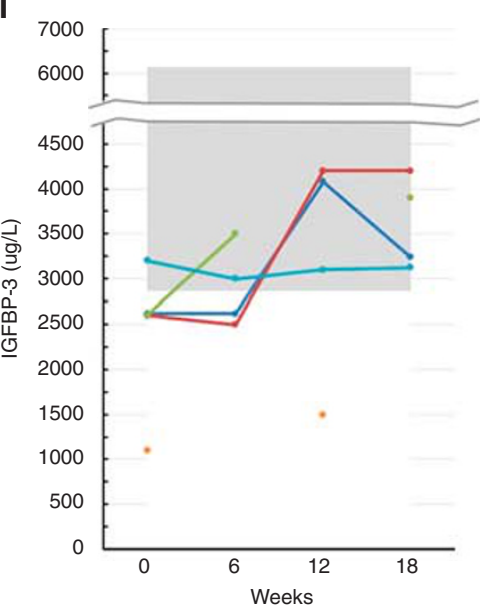

Figure 1 Effect of D-gal supplementation. Effects on (a) aspartate transaminase (AST) and (b) alanine transaminase (ALT), on (c) anticoagulation and (d-f) coagulation, and on (g) TSH, (h) TBG, and (i) IGFBP-3. The shadowed area represents the reference range. 
fell below baseline. Patient 2 developed an intercurrent illness and discontinued galactose supplementation for 2 weeks between the 12- and 18-week time point. His AST spiked upwards, almost reaching baseline at 18 weeks.

Unlike AST, ALT normalized in several patients (Figure 1b). Baseline ALT was abnormal in 5 patients (patients 1, 2, 4, 5, and 7); the rest exhibited normal ALT throughout the study. Among the 5 patients with abnormal baseline ALT, patients 1, 2, 4, and 7 normalized at 6 or 12 weeks. Except for patient 2, ALT remained normalized and stable at 12 and 18 weeks. While ALT in patient 2 normalized at 6 and 12 weeks, at 18 weeks it spiked upwards and exceeded baseline by $18 \%$. Patient 2 had an intercurrent illness (see above). Patient 5's ALT decreased by $43 \%$ at 6 weeks and remained stable at 12 and 18 weeks, at 3\% above the upper limit of normal.

ALT levels were found to be significantly decreased from T0 to T2 during the trial $(p=0.05)$. A dosage of $1.5 \mathrm{~g} / \mathrm{kg} /$ day in the last 6 weeks of the trial did not lead to significant improvement of the laboratory parameters compared to those at $1 \mathrm{~g} / \mathrm{kg} /$ day.

Improvement and normalization of coagulation parameters Anticoagulation data was available for patients 1, 2, 3, 5, 6, and 9 (Figure 1c). Only patient 9 had a normal baseline. ATIII normalized in patients 1 and 2 at 12 weeks. Patient 1 remained within normal range at 18 weeks. While patient 2's ATIII dropped $30 \%$ following illness and cessation of galactose intake, ATIII was maintained at twofold higher than baseline at 18 weeks. Patients 3 and 6 improved by $25 \%$ to $50 \%$ at 18 weeks, but did not normalize. Patient 5 transiently normalized at 6 weeks, but fell $8 \%$ below the lower limit of normal at 12 and 18 weeks.

With the inclusion of all patient data for ATIII there was a significant difference between $\mathrm{T} 0$ and $\mathrm{T} 3$ data point for ATIII $(\mathrm{P}=0.020)$

The aPTT baseline was normal in patients $2,3,5,6$, and remained stable during the study (Figure 1d). aPTT baseline was abnormal in patients 1 and 7 and normalized at 6 and 12 weeks, respectively.

Factor IX data was available for patients 1, 2, 5, 6, and 9, and the baseline was normal (Figure 1e). Factor IX levels remained normal following 18 weeks of galactose supplementation.

Other coagulation parameters, factor XI and factor XIII, normalized in patient 1 after 12-18 weeks of galactose supplementation (Figure 1f, Supplementary Figure Sb).

\section{Improvement in glycoproteins TSH, TBG, and IGFBP-3}

TSH data is available for all patients except patient 7 (Figure 1g). Patients 2 and 5 had abnormal baseline. Patient 2 normalized at 18 weeks while patient 5 normalized at 6 weeks and remained stable at 12 and 18 weeks.

TBG data is available for patients $1,2,5,6$, and 9 (Figure 1h). All patients had abnormal baseline, except for patient 5. Patients 1 and 2 normalized at 6 weeks and remained stable at 12 and 18 weeks. Patient 6 improved by $30 \%$ at 12 weeks, only $8 \%$ below the lower limit of normal. Patient 9's baseline was twofold the upper limit of normal. Although his TBG level declined by $12 \%$ at 18 weeks, it was still $80 \%$ higher than the upper limit of normal.

IGFBP-3 data is available for patients 1, 2, 3, 5, and 6 (Figure 1i). Except for patient 5, the baselines were all abnormal, ranging from $8 \%$ to $60 \%$ below the lower limit of normal. Patients 1 and 2 normalized at 12 weeks and remained stable at 18 weeks. The 12 -week time point data was missing for patient 3 ; nevertheless it normalized at 18 weeks. Patient 6 showed slight improvement at 12 weeks but remained about $50 \%$ below the lower limit of normal (Supplementary Figure Sb).

\section{Fluctuating and abnormal levels of serum creatine kinase and glucose}

Creatine kinase and glucose levels fluctuated and remained abnormal during the study. Despite severe elevation of creatine kinase, none of the patients experienced clinical rhabdomyolysis while on galactose supplementation. The frequency of hypoglycemic episodes decreased significantly in four patients. Patients 1 and 6 had recurrent hypoglycemic episodes upon fasting. Patient 5 remained on diazoxide treatment due to hyperinsulinism (data not shown).

\section{Improvement of serum transferrin glycosylation in 8 patients}

Isoelectric focusing and high-resolution mass spectrometry of intact transferrin confirmed the characteristic mixed type I/II pattern with increased fractions of both truncated glycans and lack of whole glycans in all patients' sera. Clear improvement of transferrin glycosylation was seen in 8 patients, with patterns shifting from a combined type I/II pattern to a mild type I pattern. No change was observed in patient 5 . None of the patients showed a fully normalized pattern at T3 (Supplementary Files, Supplementary Table 3, Figure 3).

The most abundant peaks in the transferrin mass spectrometry profiles were annotated, and ratios were calculated for the nonglycosylated (A-Glyco), monoglycosylated (MonoGlyco), and trisialo-transferrin (Trisialo-Glyco) over the normal transferrin peak (Di-Glyco). Ratios were compared in patient samples before D-gal supplementation and 18 weeks after starting oral supplements according to protocol. An example is shown in Figure 2 for a severely (patient 2) and a mildly (patient 9) abnormal profile. A-Glyco- and MonoGlyco ratios were abnormal in all patients. All patients except patient 5 showed significant improvement on D-gal supplementation (Figure 3). The most pronounced improvement was measured in patient 4 . In patients 3 and 8 , the A-Glyco peak normalized (Supplementary Table 3, Figure 3).

\section{Long-term monitoring of patient 1}

Patient 1 remained on galactose therapy at $1 \mathrm{~g} / \mathrm{kg} /$ day for an additional 12 months after the study ended. The parameters that improved and normalized during the study period, 

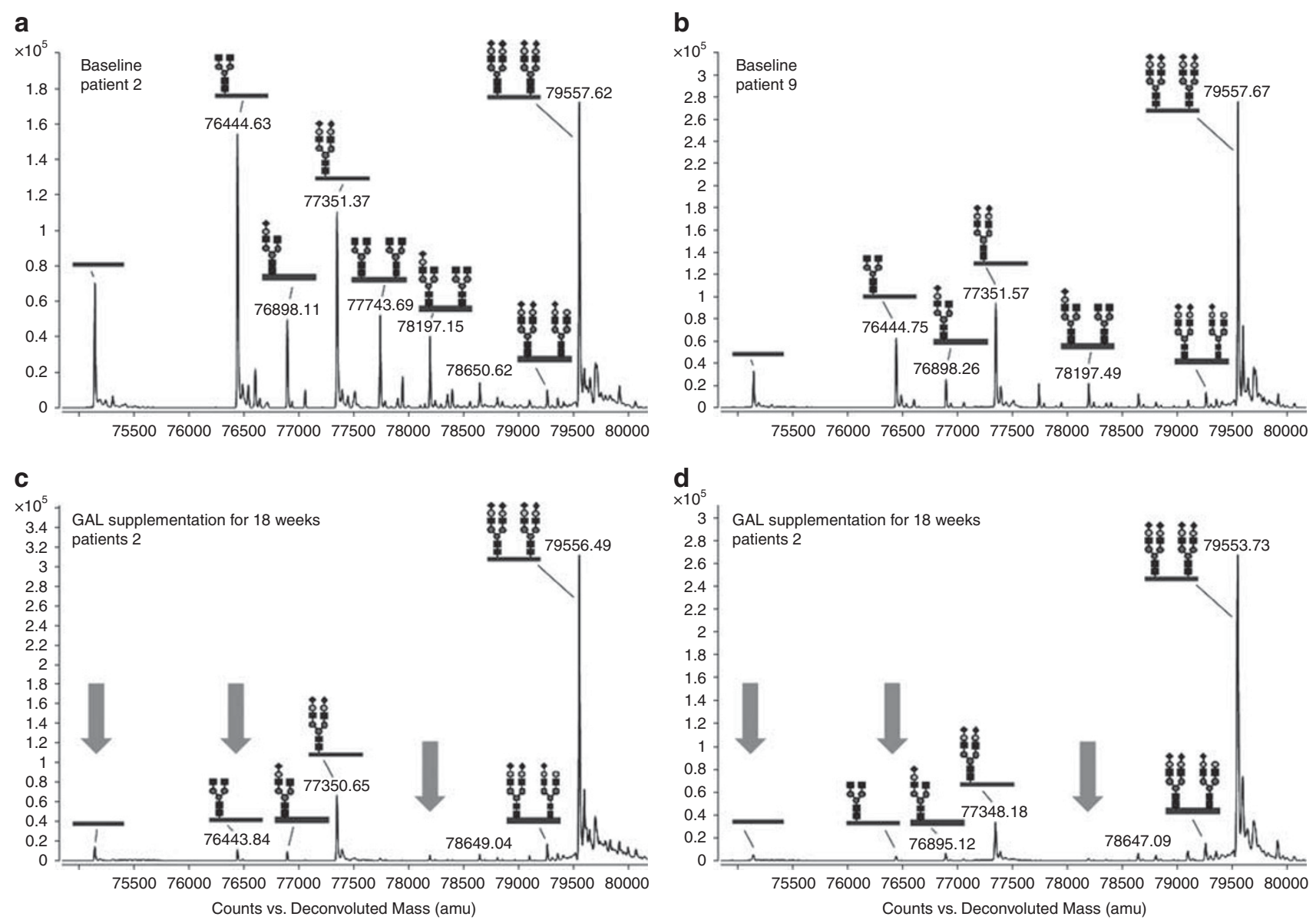

Figure 2 High-resolution mass spectrometry of intact serum transferrin. Baseline profiles of (a) patient 2 and (b) patient 9 show characteristic PGM1 glycoforms with truncated glycans and lack of whole glycans. Patient 9 shows a milder profile than patient 2; spectra of (c) patient 2 and (d) patient 9 show large improvement through the reduction of abnormal glycosylation peaks upon 18 weeks of galactose treatment, as is highlighted by the green arrows.

including ALT, aPTT, ATIII, TSH, and TBG, remained stable (Supplementary Table 2). However, factor XI and factor XIII returned to before-treatment levels. Surprisingly, creatine kinase, which remained high during the study (five-fold the upper limit of normal), decreased by almost $80 \%$ over the course of one year, falling to the near upper limit of normal.

\section{In vitro studies in PGM1-CDG skin fibroblasts}

PGM1 protein expression was moderately reduced in patient 2, who carries heterozygous missense mutations (T337M and $\mathrm{R} 503 \mathrm{Q})$. No protein was visible in patient 1 (R422W and Q530X) and cell-line 2015X (G382Vfs ${ }^{\star}$ ) (Supplementary Figure S1a).

ICAM-1 protein expression was markedly diminished in all four patients and an up to twofold increase in ICAM-1 was observed in patients 1, 2, and cell-line 2015X following galactose treatment. No improvement was detected in patient 5 (Supplementary Files, Supplementary Figure 1).

Glycomics showed that both asialylated and monosialylated biantennary $\mathrm{N}$-glycan species were elevated ( $>2$ STD from control mean) in $\mathrm{N}$-glycan profiles of total glycoprotein isolated from the cultured skin fibroblasts of patient 2 . Following galactose supplementation, asialylated biantennary glycan (Hexose5HexNAc4) at $m / z 2070.06$ was reduced to normal level. Monosialylated glycans (siallhexose5HexNAc3) at $m / z 2605+2431$, while far from normalized, showed a slight reduction (Supplementary Table $\mathbf{4 a )}$ ).

Unexpectedly, altered $O$-glycosylation was detected in patient 2's cultured skin fibroblasts, with decreased disialyl core 1 glycans at $m / z 1256$ and increased disialyl core 2 at $1706 \mathrm{~m} / z$. These alterations improved but did not completely normalize with galactose supplementation. Interestingly, galactose supplementation increased monosialo core 1 at $\mathrm{m} / \mathrm{z} 895$ both in control and PGM1-CDG cells (Supplementary Table $4 b$ ).

LLO analysis detected a large amount of shortened LLO in all four patient cells ( $\mathrm{Man}_{9} \mathrm{GlcNAc}_{2}$-PP-dolichol). Galactose supplementation led to the reduction of shortened LLO ( $\mathrm{Man}_{9} \mathrm{GlcNAc}_{2}$-PP-dolichol) in patients 1, 2, and cell-line 2013Y, resulting in a LLO profile similar to control. No 

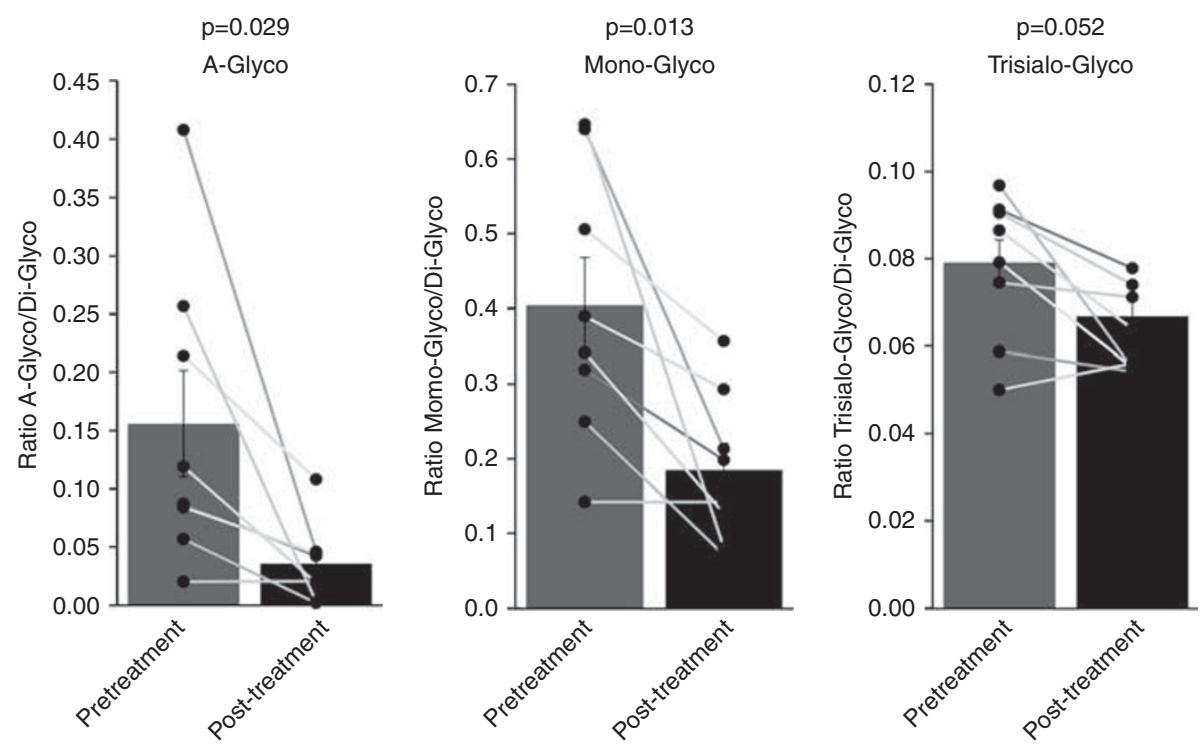

Figure 3 High-resolution transferrin glycosylation analysis in PGM1-CDG patients before and after D-gal supplement. Ratios of a-, mono-, and tri-sialo over tetra-sialo transferrin were calculated and compared with references ranges (repeated measures of analysis of variance). A-Glyco: $\leq 0.01040$, Mono-Glyco: $\leq 0.02700$ Trisialo-Glyco: $\leq 0.031900$.

improvement was observed in patient 8 . In none of the patients did D-gal supplementation have an effect on the PLO profile (Figure 4, LLO top and bottom row).

Because the synthesis of the sugar donor substrate, dolichylphosphate-glucose (Dol-P-Glc), requires UDP-Glc, we hypothesized that PGM1 deficiency would disrupt normal glucose and galactose metabolism, leading to a disbalance of available nucleotide sugars for Dol-P-Glc synthesis and resulting in the observed accumulation of shortened LLO ( $\mathrm{Man}_{9} \mathrm{GlcNAc}_{2}$-PP-dolichol). In patient cell lines 1, 2, 8, and cell-line 2013Y, we found increased levels of UDP-Glc $(72.2 \%$ and $49.3 \%$ of control, $141.3 \%$ and $90.5 \%$ of control, $60.9 \%$ and $23.0 \%$ of control, and $142.1 \%$ and $87.7 \%$ of control, respectively) and in a lesser degree UDP-Gal $(29.6 \%$ and $23.4 \%$ of control, $84.4 \% 69.7 \%$ of control, $9.1 \%$ and $-22 \%$ of control, $48.3 \%$ and $32.7 \%$ of control, respectively), in the presence of glucose in the medium. D-Gal supplementation increased the levels of both UDP-Glc and UDP-Gal. The same effect in control cells occurred on the first day of D-gal supplements but normalized at 4 days. In all patient cell lines both UDP-Glc and UDP-Gal remained significantly increased on 4 days of D-gal supplementation. Nucleotide sugar ratios UDP-Glc/UDP-Gal in controls were at a mean of 1.2 , as previously reported, ${ }^{3}$ both with or without D-gal, while in patient cell lines at mean 1.63 , they were similar to that on D-gal (1.56); suggesting that adding D-gal didn't restore the nucleotide sugar ratio (Supplementary Table 5).

\section{DISCUSSION}

\section{Clinical and laboratory improvement in patients}

There is no proven effective treatment available in most CDG types, except for mannosephosphate isomerase deficiency (MPI-CDG), where coagulopathy and hypoglycemia are successfully treated by oral mannose supplementation. ${ }^{14-16}$ Based on a hypogalactosylation glycanpattern in PGM1-CDG, compassionate use of $\mathrm{D}$-gal resolved hypogonadotropic hypogonadism in two patients. ${ }^{3}$ In the present prospective pilot study, we assessed different laboratory parameters as potentially secondary endpoint markers in blood. Those patients's parameters, which were abnormal at baseline, improved or normalized by 12 or 18 weeks, with a few exceptions. ALT was abnormal at baseline in half of the patients and completely normalized in all but one patient. Interestingly during infection-related diarrhea in patient 2 the liver transaminases dramatically reincreased within 2 weeks. AST was abnormal in all patients. Although AST levels markedly improved in most patients on D-gal, none normalized during the study period. AST is also a biomarker for muscle involvement. Creatine kinase, another biomarker of muscle injury, also remained elevated in all patients. Persistently elevated AST might be indicative of unresolved myopathy, caused by insufficient glycogenolysis. ${ }^{17}$ The observed improvement in both serum transaminases suggests that galactose supplementation ameliorates liver dysfunction, thus decreasing the risk for hepatic steatosis and fibrosis. We also demonstrated that D-gal supplementation was safe and tolerated at $1.5 \mathrm{~g} / \mathrm{kg} /$ day, even using $50 \mathrm{~g} / \mathrm{kg} /$ day (patients 1,2 , and 3). ATIII also improved and was found to be significantly higher in patients on D-gal. ATIII could be a potential biomarker for future treatment trials.

Long-term use of D-galactose in patient 1 over one year beyond the study period showed normalization of almost all biochemical anomalies, except for transferrin glycosylation. Normalization of several coagulation factors on galactose resolved the patient's frequent, spontaneous epistaxis episodes, and improved quality of life. Some of the coagulation 



Figure 4 Lipid-linked oligosaccharide analysis in 4 PGM1 deficient cell lines showing reduced LLO at baseline and improvement following D-gal supplementation. To investigate whether PGM1 deficiency disrupts the formation of LLO, which is a required precursor for the synthesis of nascent $N$-linked glycoproteins in the ER, we performed lipid-linked oligosaccharide (LLO) and protein-linked oligosaccharide (PLO) analyses in the skin fibroblasts of patients 1, 2, 8, and cell-line 2013Y. The cells were deprived of glucose while the culture media was supplemented without or with $10 \mathrm{mM}$ D-gal (for 1 hour). The level of full-length LLO (Glc ${ }_{3} \mathrm{Man}_{9} \mathrm{GlCNAC}_{2}-\mathrm{PP}-\mathrm{Dol}, \mathrm{G} 3$ ) or sugar moieties bound to newly synthesized PLO (mainly $\mathrm{Glc}_{1} \mathrm{Man}_{9}$ and $\mathrm{Man}_{9}$ ), remained fairly unchanged in control cells (Figure 4, left column), indicating a high degree of metabolic fitness in these cells. In contrast, cells from all four patients showed a large amount of shortened LLO (Man, GlcNAc2-PP-dolichol) (Figure 4, LLO top row). In contrast, the PLO profile was indistinguishable from the control cells (Figure 4, PLO top row). Interestingly, galactose supplementation led to the reduction of shortened LLO (Man $G_{9}$ ICNAC $_{2}$-PP-dolichol) in patients 1, 2, and cell-line 2013Y, resulting in a LLO profile that is similar to control. No improvement was observed in patient 8 (Figure 4, LLO bottom row). D-Gal supplementation had no effect on the PLO profile in all patients (Figure 4, PLO bottom row). HPLC, highperformance liquid chromatography.

factors remained abnormal, which might suggest the need for either higher D-gal dose or longer treatment and follow-up. Interestingly, the frequency of hypoglycemia and fluctuation of creatine kinase levels also decreased, without episodes of rhabdomyolysis.

\section{Improved glycosylation in patients}

Serum transferrin glycosylation showed a characteristic PGM1 mixed type I/type II profile in all patients at baseline, with truncated glycans and lack of whole glycans. Upon galactose supplementation, the spectrum shifted from a mixed profile to a mild type I profile. Only patient 5 did not shift to a type I glycosylation, but this patient was also on the mildest spectrum of all patients. The spectra did not completely normalize; mostly type I peaks remained present. However, most of the truncated galactose-lacking glycoforms strongly decreased during this 18 weeks of supplementation. Overall, the spectra suggest that galactose is able to directly restore the lack of galactose on the truncated glycans, while there seems to be a second, unsolved mechanism of action of galactose on the slower improvement of CDG type I glycosylation, which could potentially lead to a completely normalized glycosylation.

In vitro D-gal effects on glycosylation in patient fibroblasts ICAM1 is a known cell-marker of glycosylation. In contrast to patient 5, patients 1 and 2 and cell-line 2015X showed an increase in ICAM1 expression upon in vitro galactose treatment. This was in concert with the improvement of glycomics results in fibroblasts of patient 2 on galactose. Interestingly, before in vitro galactose treatment the sialylation of $\mathrm{N}$-glycans was found to be reduced, with increased hyposialylated subspecies. Galactose treatment improved sialylation (Supplementary Files).

O-linked glycosylation in PGM1-deficient cells showed a significant reduction of disialo-corel species and increase of sialylated core2 species. Since core-2 GlcNAc-transferase, the rate-limiting step of core2 synthesis, competes with ST6GalNAc-transferase, increased sialylated core2 and core1 ratio points to a deficiency at the step of ST6GalNAc transferase that forms 2,6 sialic acid motifs on disialo-core1 $\mathrm{T}$ antigen. However, 2,3 sialylation in $\mathrm{O}$-linked glycosylation is not 
significantly affected in PGM1-CDG fibroblasts. Similar to the effect on $\mathrm{N}$-linked glycosylation, galactose treatment improved the level of disialo corel species and partially corrected the ratio between disialo core2/disialo core1. The $\mathrm{Km}$ of CMP-sialic acid for sialyltransferase of $\mathrm{O}$-linked glycosylation is at least 10 times higher than the $\mathrm{Km}$ for sialyltransferases of $\mathrm{N}$-linked glycosylation. Thus, it is more likely that the selective difference between 2,3 sialylation and 2,6 sialylation in $O$-GalNAc glycosylation is related to the difference in $\mathrm{Km}$ of these different sialyltransferases for CMPsialic acid. Future studies on CMP-sialic acid levels in PGM1 deficient cells is necessary to better understand the changes in O-linked glycosylation.

\section{In vitro galactose effects on lipid-linked oligosaccharides and nucleotide sugars in patient fibroblasts}

LLO analysis on serum starvation revealed an accumulation of shortened LLO in cultured patient skin fibroblasts. This indicates a disruption in the synthesis of LLO, required for $\mathrm{N}$-linked glycosylation in the ER. Interestingly, accumulation of $\mathrm{Man}_{9} \mathrm{GlcNAc}_{2}$-PP-Dol is characteristic for several type I CDGs, including ALG6-CDG, ${ }^{18}$ ALG8-CDG,${ }^{19}$ and MPDU1CDG. $^{20,21}$ Whereas the activity of glucosyltransferases is impaired in the first two CDG subtypes, the synthesis of Dol$\mathrm{P}$-Glc is defective in the latter case. PGM1 deficiency, in the lack of normal nutrients, like in the experimental setup of serum starvation, depleted the cellular pool of UPD-Glc, available for Dol-P-Glc synthesis (data not shown). Interestingly, both UDP-Gal and UDP-Glc, depleted in patient cells during serum starvation, normalized on D-gal supplements (data not shown). Also the LLO profile restored in patient cell-line 2013Y, strongly suggesting a link between PGM1, glucose metabolism, and $\mathrm{N}$-glycosylation. D-gal supplementation did not improve LLO in patient 8, who is severely PGM1deficient. UDP-Gal and particularly UDP-Glc might have been diverted towards energy production and not used for Dol-P-Glc synthesis in this case.

In the presence of sufficient glucose in the culture medium during the experiments, UDP-Glc and UDP-Gal pools were elevated in patient's cells (Supplementary Table 5). D-gal treatment led to a further increase in nucleotide sugar levels of UDP-Glc and UDP-Gal in patients, but did not change the UDP-Glc to UDP-gal ratio. Controls showed similar changes but normalized after 4 days. Based on our experiments, in spite of increasing nucleotide sugar concentrations on D-gal, the ratio of UDP-Glc/UDP-Gal didn't change, therefore still affected substrate availability for glycosylation. This means that the D-gal related UDP-monosaccharide pool improvement cannot be the only explanation for the improvement of glycosylation, and for the clinical improvement in patients, and we should further search for other regulatory mechanisms.

\section{Limitations}

Patients with PGM1-CDG show diverse abnormalities in endocrine, coagulation, and liver function tests; therefore, our study included inconsistent baseline laboratory values. Noncompliance was observed in one patient, one patient accidentally prematurely increased D-gal dose, and a gastrointestinal infection affected treatment in another patient. Another limitation of this study was the small sample size, wide spectrum of ages at the time of enrollment (ranging from 19 months to 21 years), and the lack of adequate handling or volume of some of the blood samples at various time points.

Statistical analysis was reliable only for the serum transferrin samples, where all baseline levels were abnormal, and for ATIII, showing significant improvement on D-gal. Other laboratory values, showing frequent normal values even spontaneously during disease course, were not reliable markers for end points, and for statistical analysis.

\section{CONCLUSIONS}

Here we demonstrated easy and safe administration of oral D-gal and quick, significant clinical laboratory and metabolic improvements in patients with PGM1-CDG.

The in vitro detected $\mathrm{N}$-glycan hyposialylation, altered $O$-linked glycans and abnormal LLO-profile in patientfibroblasts showed improvement or normalization following D-gal treatment, in concert with improvement in the glycome, liver function, and coagulation in D-gal treated patients. Transferrin isoforms, ALT, and ATIII could be end-point markers for future clinical trials in PGM1-CDG.

We suggest using oral D-gal as medical food in PGM1-CDG. An 18 -week escalating dose protocol up to $1.5 \mathrm{~g} / \mathrm{kg} /$ day was proved to be safe and effective in our prospective pilot study. We should emphasize that further research is needed, with objective, placebo controlled trials, especially for optimizing long-term therapy.

\section{SUPPLEMENTARY MATERIAL}

Supplementary material is linked to the online version of the paper at http://www.nature.com/gim

\section{ACKNOWLEDGMENTS}

This study is supported in part by the Hayward Foundation and by 1 U54 GM104940 from the National Institute of General Medical Sciences of the National Institutes of Health, which funds the Louisiana Clinical and Translational Science Center. Additional support comes from the European Union's Horizon 2020 research and innovation program under the ERA-Net for Research on Rare Diseases, from the Netherlands Organisation for Scientific Research (ZONMW Medium Investment grant 40-00506-98-9001 and VIDI grant 91713359 to D.J.L., VENI grant 016168079 to M.v.S.) and the Prinses Beatrix Spierfonds (grant W.OR15-16 to D.J.L. and M.v.S.). T.H. and H.H. was supported by General University Hospital in Prague, Czech Republic (RVO-VFN 64165), and the Ministry of Health of the Czech Republic (MZ CR AZV 16-31932A). We thank the patients and their families for their participation. EM and CT this work was supported by the European Union's Horizon 2020 research and innovation program under the ERA-NET Cofund action N N 643578 EURO-CDG-2 and for CT sustained by the German 
Bundesministerium fuer Bildung und Forschung under the frame of E-Rare-3. We are also grateful to Hudson Freeze at the Burnham Institute (San Diego, CA) for sharing a patient cell line with us. We also thank for the trial support by Jos Dederen.

\section{DISCLOSURE}

The authors declare no conflict of interest.

\section{REFERENCES}

1. Jaeken J. Congenital disorders of glycosylation (CDG): it's (nearly) all in it!. J Inherit Metab Dis 2011;34:853-858.

2. Timal S, Hoischen A, Lehle L, et al. Gene identification in the congenital disorders of glycosylation type I by whole-exome sequencing. Hum $\mathrm{Mol}$ Genet 2012;21:4151-4161.

3. Tegtmeyer LC, Rust S, van Scherpenzeel M, et al. Multiple phenotypes in phosphoglucomutase 1 deficiency. N Engl J Med 2014;370:533-542.

4. Wong SY-W, Beamer LJ, Gadomski T, et al. Defining the phenotype and assessing severity in phosphoglucomutase-1 deficiency. J Pediatr 2016;175:130-136.e8.

5. Morava E. Galactose supplementation in phosphoglucomutase-1 deficiency; review and outlook for a novel treatable CDG. Mol Genet Metab 2014:112:275-279.

6. Stojkovic T, Vissing J, Petit F, et al. Muscle glycogenosis due to phosphoglucomutase 1 deficiency. N Engl J Med 2009;361:425-427.

7. Beamer LJ. Mutations in hereditary phosphoglucomutase 1 deficiency map to key regions of enzyme structure and function. J Inherit Metab Dis 2015;38:243-256.

8. Lee $Y$, Stiers KM, Kain BN, et al. Compromised catalysis and potential folding defects in in vitro studies of missense mutants associated with hereditary phosphoglucomutase 1 deficiency. J Biol Chem 2014;289: 32010-32019.

9. De Smet E, Rioux J-P, Ammann H, et al. FSGS permeability factorassociated nephrotic syndrome: remission after oral galactose therapy. Nephrol Dial Transplant 2009;24:2938-2940.

10. van Scherpenzeel M, Steenbergen G, Morava E, et al. High-resolution mass spectrometry glycoprofiling of intact transferrin for diagnosis and subtype identification in the congenital disorders of glycosylation. Trans/ Res 2015;166:639-649.e1.
11. Thiel C, Schwarz M, Peng J, et al. A new type of congenital disorders of glycosylation (CDG-li) provides new insights into the early steps of dolichol-linked oligosaccharide biosynthesis. J Biol Chem 2003;278: 22498-22505.

12. Kochanowski N, Blanchard F, Cacan R, et al. Intracellular nucleotide and nucleotide sugar contents of cultured $\mathrm{CHO}$ cells determined by a fast, sensitive, and high-resolution ion-pair RP-HPLC. Anal Biochem 2006;348: 243-251.

13. Xia B, Zhang W, Li X, et al. Serum N-glycan and O-glycan analysis by mass spectrometry for diagnosis of congenital disorders of glycosylation. Anal Biochem 2013;442:178-185.

14. Niehues R, Hasilik M, Alton G, et al. Carbohydrate-deficient glycoprotein syndrome type $\mathrm{lb}$. Phosphomannose isomerase deficiency and mannose therapy. J Clin Invest 1998;101:1414-1420.

15. Ichikawa M, Scott DA, Losfeld $M-E$, et al. The metabolic origins of mannose in glycoproteins. J Biol Chem 2014;289:6751-6761.

16. Freeze HH. Perhaps a wee bit of sugar would help. Nat Genet 2016;48: 705-707.

17. Scott K, Gadomski T, Kozicz T, et al. Congenital disorders of glycosylation: new defects and still counting. J Inherit Metab Dis 2014;37:609-617.

18. Körner $C$, Knauer $R$, Holzbach $U$, et al. Carbohydrate-deficient glycoprotein syndrome type $\mathrm{V}$ : deficiency of dolichyl-P-Glc: Man9GlcNAc2-PP-dolichyl glucosyltransferase. Proc Natl Acad Sci USA 1998;95:13200-13205.

19. Chantret I, Dancourt J, Dupré T, et al. A deficiency in dolichyl-P-glucose: Glc1Man9GIcNAc2-PP-dolichyl alpha3-glucosyltransferase defines a new subtype of congenital disorders of glycosylation. J Biol Chem 2003;278: 9962-9971.

20. Kranz C, Denecke J, Lehrman MA, et al. A mutation in the human MPDU1 gene causes congenital disorder of glycosylation type If (CDG-If). J Clin Invest 2001;108:1613-1619.

21. Schenk B, Imbach T, Frank CG, et al. MPDU1 mutations underlie a novel human congenital disorder of glycosylation, designated type If. J Clin Invest 2001;108:1687-1695.

22. Maliekal P, Sokolova T, Vertommen D, et al. Molecular identification of mammalian phosphopentomutase and glucose-1,6-bisphosphate synthase, two members of the alpha-D-phosphohexomutase family. J Biol Chem 2007;282:31844-31851.

23. Ondruskova N, Honzik T, Vondrackova A, et al. Glycogen storage disease-like phenotype with central nervous system involvement in a PGM1-CDG patient. Neuro Endocrinol Lett 2014;35:137-141. 